\title{
Prognostic Evaluation of Vitamin D Deficiency in Breast Cancer Patients: A Pilot Study in India
}

\author{
Bela Goyal ${ }^{1}$ and Sanjeev Garg ${ }^{2 *}$ \\ 'Department of Biochemistry, Govt. Medical College and Hospital, Sector-32, Chandigarh, India \\ ${ }^{2}$ Department of Pathology, Grecian superspeciality Cancer hospital, Mohali, India
}

\section{ABSTRACT}

Background: Vitamin D deficiency has been associated with poor prognosis in breast cancer. A new molecular classification of breast cancer is being increasingly used that is based on expression of estrogen receptors, progesterone receptors and Her $2 /$ neu amplification and is found to correlate well with prognosis and outcome of disease. The present study was designed to determine serum vitamin D deficiency in these molecular subtypes of breast cancer.

Methods: Estimation ofserum vitamin D levels by chemiluminiscence was done in a cohort of 99 breast cancer patients and 25 age matched healthy controls. Breast cancer patients were further grouped into luminal A, luminal B, Her2/neu enriched and triple negative subtypes based on immunohistochemistry results. Non-parametric tests were performed to compare vitamin D levels in different groups.

Results: $54.6 \%, 60 \%, 66.6 \%$ and $84.2 \%$ of patients with luminal A, luminal B, Her $2 /$ neu enriched and triple negative subtypes respectively were found to be vitamin D deficient $(<20 \mathrm{ng} / \mathrm{mL})$ as compared to only $20 \%$ in healthy controls. More aggressive Her $2 /$ neu enriched and triple negative subtype patients had significantly lower serum vitamin D levels than the luminal subtypes of breast cancer.

Conclusion: Vitamin D deficiency is more prevalent and significantly lower levels are found in more aggressive subtypes of breast cancer. Vitamin D supplementation must be considered in these forms of breast cancer to improve outcome.

Keywords: Vitamin D Deficiency, Breast Cancer, Her2neu Enriched Breast Cancer, Triple Negative Breast Cancer

\section{Introduction}

In addition to maintaining calcium and phosphate homeostasis, vitamin $\mathrm{D}$ has been proposed to have antiproliferative effects on various cancer subtypes. It is believed to retard tumor cell growth and proliferation, promote apoptosis, prevent tumor cell stimulation by growth factors and regulate inflammation, thus affecting tumor microenvironment ${ }^{[1]}$. Low levels of vitamin $\mathrm{D}$ have been shown to be associated with increased mortality and poor prognosis in breast cancer in various studies in different parts of the world..$^{[2,3,4]}$

Breast cancer is the most common cancer among Indian urban females. ${ }^{[5]}$ It has classically been classified based on morphologyor histological grade into various types taking into account tumor cell's mitotic index, degree of differentiation and nuclear pleomorphism. ${ }^{[6]}$ However, within morphological and histological subtypes, variation was observed in terms of prognosis and response to therapy. With the advent of microarray based gene expression profiling, various classification systems emerged. Recently, a microarray based molecular classification proposed by Perou and colleagues ${ }^{[7]}$ and validated by others has become the most acceptable classification to determine prognosis. It classifies the breast cancer into luminal A, luminal B, Her2neu enriched and basal type/ triple negative breast cancer (TNBC) based on the tumor cells expression of estrogen receptor(ER), progesterone receptor (PR) or Her2/neu amplification. The status of ER, PR and Her2neu expression is routinely determined by immunohistochemistry and fluorescence in situ hybridization(FISH) ${ }^{[8]}$

Based on a case study in California, that has shown deficient levels of vitamin $\mathrm{D}$ in breast cancer patients subtypes according to the above mentioned classification, ${ }^{[9]}$ the present study was planned to determine vitamin D levels in the breast cancer patients as per the molecular classification, to compare the serum vitamin D levels among the various groups of breast cancer patients and also with age matched healthy controls in India.

\section{Materials and Methods}

A consecutive case series study was conducted at a tertiary care hospital in North India in the year 2012-2014. Blood samples of 99 newly diagnosed breast cancer patients 
presenting to the hospital were collected in vacutainers. Blood was allowed to clot for 20-30 min and centrifuged at $3000 \mathrm{Xg}$ for $20 \mathrm{~min}$. to separate the serum.

Serum total Vitamin D estimation was performed by fully automated chemiluminescence based autoanalyser, Advia centaur XP (Siemens, USA). The performance of the instrument and quality controls were satisfactory before analyzing the samples.

The cohort of breast cancer patients was divided into 4 groups based on the immunohistochemical analysis on breast cancer cells. Luminal A group showed positivity for Estrogen Receptors (ER) and/or Progesterone receptors (PR) and were negative for Her2/neu overexpression. Luminal B group showed positivity with ER, PR as well as Her2/neu overexpression. Her2/neu enriched group showed Her2/neu overexpression but negative for ER and PR. Triple negative breast cancer (TNBC) group showed negative status with ER,PR as well as Her2/neu.

Total 25 voluntary healthy controls were also enrolled in the study and their serum vitamin D levels were measured. Based on the serum vitamin D levels, these groups were subdivided into vitamin $\mathrm{D}$ deficient (vitamin $\mathrm{D} \leq 20 \mathrm{ng}$ / $\mathrm{mL}$ ), vitamin D insufficient (vitamin D between 20-29 ng/ $\mathrm{mL}$ ), and vitamin $\mathrm{D}$ sufficient (vitamin $\mathrm{D} \geq 30 \mathrm{ng} / \mathrm{mL}$ ). ${ }^{[10]}$

Statistical analysis was performed using SPSS v.16 (SPSS, USA). Non-parametric Mann-Whitney U test was carried out to compare the various groups of breast cancer patients with healthy controls. Unpaired student t- test was performed to match the age of breast cancer patients and healthy controls. Non-parametric kruskal- wallis test followed by Mann-Whitney U test was done to compare these groups with each other. P value $<0.05$ was considered statistically significant.

\section{Results}

The mean age of patients with breast cancer was found to be 55.8 with standard deviation of 10.09 . Table 1 shows the sample size, mean, standard deviation and $95 \%$ confidence interval of serum vitamin D levels of all the breast cancer patients groups and healthy controls.

The mean serum vitamin D levels in all the groups of breast cancer patients was found to be significantly lower than the healthy controls with $\mathrm{p}$ value $<0.05$ as shown in table 2 and figure 1. Furthermore, serum vitamin D levels in Her2/ neu enriched group and Triple negative breast cancer group were significantly lower $(p<0.05)$ than vitamin $D$ levels in the sera of Luminal A and B type of breast cancer patients.

On subdividing the groups based on the levels of vitamin $\mathrm{D}$, it was observed that luminal A type breast cancer patients had $54.6 \%(\mathrm{n}=35)$ patients with deficient vitamin $\mathrm{D}, 32.8 \%(\mathrm{n}=21)$ had insufficient serum vitamin $\mathrm{D}$ levels and only $12.5 \%(n=8)$ were having sufficient vitamin $D$ levels. Luminal B type breast cancer patients group had $60 \%(\mathrm{n}=6), 30 \%(\mathrm{n}=3)$ and $10 \%(\mathrm{n}=1)$ patients showing deficient, insufficient and sufficient levels of serum vitamin D levels respectively. However, in the Her2/neu enriched and TNBC type breast cancer patients, none of the patients showed sufficient serum vitamin D levels. Rather $66.6 \%(n=4)$ of Her $2 /$ neu enriched and $84.2 \%(n=16)$ of TNBC patients were deficient in vitamin D levels. This is in contrast to the healthy controls group where only $20 \%(\mathrm{n}=5)$ were deficient in vitamin D levels, whereas, $48 \%(n=12)$ and $32 \%(n=8)$ of the total subjects showed insufficient and sufficient levels of serum vitamin D levels as also depicted in figure 2 . This clearly shows that vitamin $\mathrm{D}$ deficiency is more prevalent in breast cancer patients as compared to age matched healthy controls with Her2/neu enriched and TNBC patients showing even lower values of serum vitamin $\mathrm{D}$ than in luminal types of breast cancer.

Table1: Mean, SD, 95\% Confidence interval of serum Vitamin D levels for Breast cancer patients groups and Healthy controls.

\begin{tabular}{|l|c|c|c|}
\hline Groups & Sample size & Mean \pm SD of Vitamin D $(\mathbf{n g} / \mathbf{m L})$ & $\mathbf{9 5 \%}$ Confidence interval \\
\hline Luminal A & 64 & $19.2 \pm 8.57$ & $17.05-21.34$ \\
\hline Luminal B & 10 & $18.9 \pm 6.52$ & $14.26-23.59$ \\
\hline Her2neu enriched & 6 & $15.7 \pm 5.98$ & $9.44-21.99$ \\
\hline Triple negative & 19 & $13.31 \pm 5.19$ & $10.8-15.81$ \\
\hline Healthy Controls & 25 & $25.95 \pm 8.09$ & $22.61-29.29$ \\
\hline
\end{tabular}

Table2: Mann whitney U test comparing Breast cancer patients groups with healthy controls.

\begin{tabular}{|l|c|c|c|c|}
\hline Groups & Mann-Whitney U & Wilcoxon W & Z value & P value \\
\hline Luminal A & 471.0 & 2.55 & -3.0 & $0.003^{*}$ \\
\hline Luminal B & 60.5 & 115.5 & -2.35 & $0.018^{*}$ \\
\hline Her2neu enhanced & 22.5 & 43.5 & -2.62 & $0.009^{*}$ \\
\hline Triple negative & 42.0 & 232.0 & -4.63 & $0.000^{*}$ \\
\hline
\end{tabular}

*indicates statistically significant at $p<0.05$ 


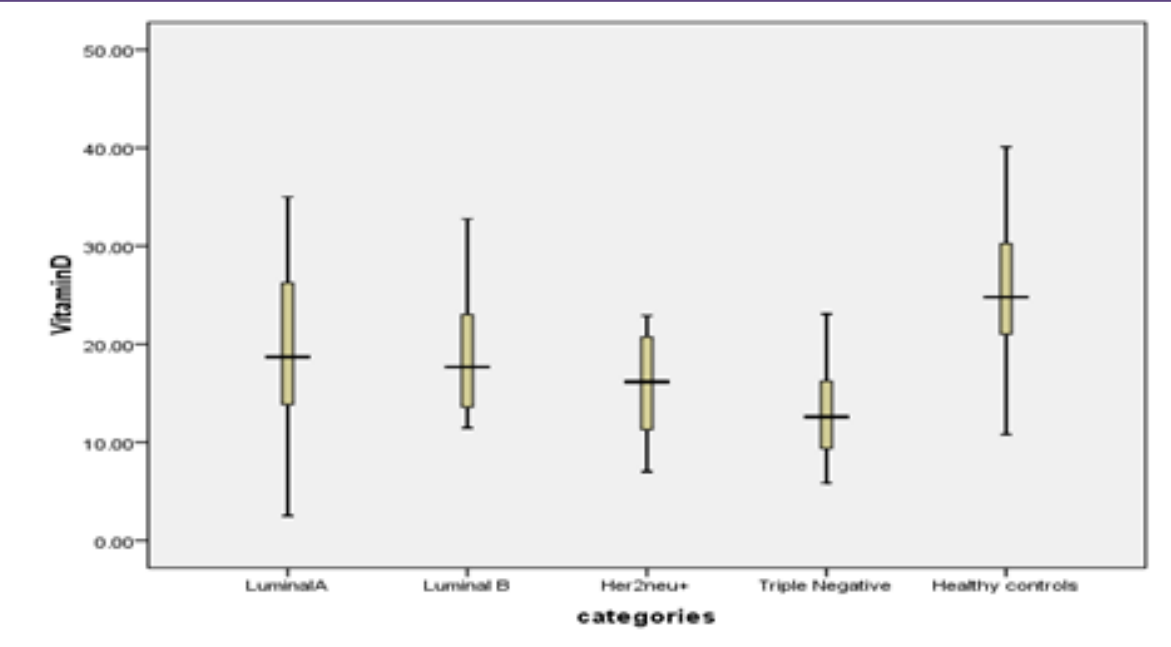

Fig. 1: Box plot showing Mean \pm SEM of Vitamin D levels (ng/ml) in Breast cancer patients groups and healthy controls. Solid bold line represents mean serum vitamin $\mathrm{D}$ in different groups.

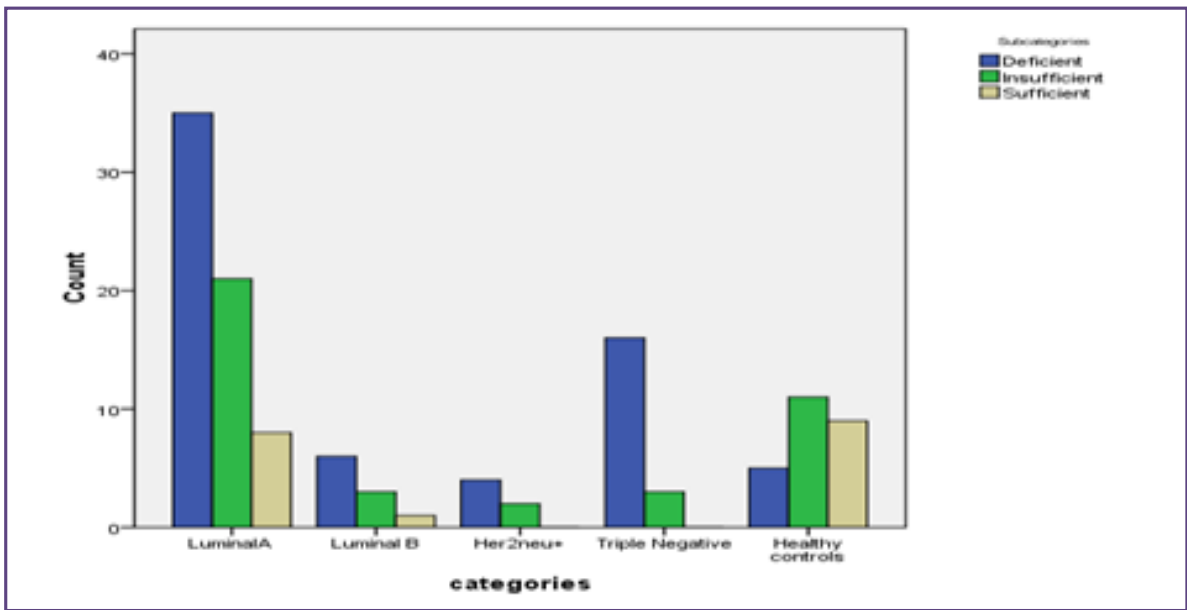

Fig. 2: Clustered Bar diagram showing number of subjects with Deficient Vitamin D levels $(\leq 20 \mathrm{ng} / \mathrm{mL})$, Insufficient Vitamin D levels(20-29ng/mL) and Sufficient Vitamin D levels $(\geq 30 \mathrm{ng} / \mathrm{mL})$ in Breast cancer patients groups and healthy controls.

\section{Discussion}

Despite the availability of a large number of studies on the role of vitamin $\mathrm{D}$ in breast cancer across the globe, the studies in India are limited. With the advent of new classification based on the receptor status in breast cancer patients, the current study was planned to look for the vitamin D deficiency in various subtypes of breast cancer.

In a country like India where skin pigmentation, milk intolerance along with lifestyle and avoidance of sun exposure already predispose to low vitamin D levels, the deficiency of vitamin $\mathrm{D}$ in breast cancer patients can result in poorer prognosis.

With a mean of $17.8 \mathrm{ng} / \mathrm{mL}$ of serum vitamin $\mathrm{D}$ in the breast cancer patients as compared to the mean of $25.9 \mathrm{ng} /$ $\mathrm{mL}$ in healthy controls, the present study shows agreement with previous studies in Saudi Arabia ${ }^{[11]}$ and in other parts of the world. ${ }^{[2,3,4]}$ The present study also shows that levels of vitamin $\mathrm{D}$ in triple negative breast cancer with mean of $13.3 \mathrm{ng} / \mathrm{mL}$ is lowest among all the subtypes. This finding conforms to the earlier studies where vitamin D levels in TNBC have been found to be lowest. ${ }^{[9]}$ Furthermore, there are studies that show that TNBC has the worst prognosis than its other counterparts ${ }^{[12]}$. Her2neu overexpression is long known to have contribution to aggressive behavior of breast cancer with poor outcomes ${ }^{[13]}$. Her2neu enriched subtype of breast cancer has been also shown to have unfavourable prognosis as compared to the luminal types. ${ }^{[14]}$ Pertaining to the anti-proliferative and protective effect of vitamin D on cancer cells, its deficiency implies higher proliferation in cancer cells and this could explain lower 
levels of vitamin D in the Her2/neu enriched and TNBC subtypes that are more aggressive and are associated with poor outcomes. Contrary to our study, a study by Kim $\mathrm{HJ}$ in korea has shown relation of vitamin D deficiency with poor prognosis of luminal types but no relation was shown with her2/neu enriched or TNBC subtype. They have hypothesized it to be due to effect of vitamin $\mathrm{D}$ on suppressing estrogen mediated proliferation of breast cancers that are ER positive i.e. luminal type but they have not explained any mechanism for ER negative cancers. ${ }^{[15]}$ However, In a recent study, it was shown that by upregulating the vitamin $\mathrm{d}$ receptors in murine model of TNBC, antiproliferative effect is enhanced ${ }^{[16]}$ and thus explaining the effect of vitamin D in TNBC subtype breast cancer. Further studies explaining the exact molecular mechanism underlying such observations needs to be carried out. Moreover, studies with larger sample size involving larger geographical distribution have to be carried out to validate our results.

Studies are also available that show that vitamin D supplementation in breast cancer patients improved their prognosis. ${ }^{[17]}$ One such study showed that intake of high dose weekly vitamin D supplementation can be helpful not only in breast cancer but also in reducing treatment related arthralgias and fractures. ${ }^{[18]}$

In the current study, a lower mean level of vitamin D was observed in both the breast cancer patients as well as healthy controls than the previous studies in western population. The plausible explanation for this could be socio-cultural taboos and vegetarian diet combined with lack of awareness regarding vitamin D supplements in Indian population as explained elsewhere. ${ }^{[10]}$ Their study also showed that mean levels of vitamin D in Punjab, Haryana are around $25.3 \mathrm{ng} / \mathrm{mL}$ which is close to the mean vitamin D levels in healthy controls $(25.95 \mathrm{ng} / \mathrm{mL})$ in our study.

The drawback of the current study includes the lower sample size, single geographical area and determination of a single parameter to evaluate prognosis in breast cancer patients. Therefore further studies considering these aspects must be carried out. However, to the best of our knowledge, this is the first study in India, evaluating role of vitamin D deficiency in breast cancer taking into account the molecular subtypes of breast cancer.

To conclude, Vitamin D deficiency is associated more with breast cancer as compared to age matched healthy controls. The Triple negative and her $2 /$ neu enriched subtypes that carry poorer prognosis shows higher prevalence and significantly lower mean values of serum vitamin $\mathrm{D}$ than the luminal types.

\section{Acknowledgement}

There is no funding or conflict of interest involved.

\section{References}

1. Moukayed M, Grant WB. Molecular link between vitamin D and cancer prevention. Nutrition. 2013;5(10):3993-4021. doi:10.3390/nu5103993.

2. Tretli S, Schwartz GG, Torjesen PA, Robsahm TE. Serum levels of 25- hydroxyvitamin D and survival in Norwegian patients with cancer of breast, colon, lung, and lymphoma: a population-based study. Cancer Causes Control ;2012: 363-370.

3. Peppone LJ, Rickles AS, Janelsins MC, Insalaco MR, Skinner KA. The association between breast cancer prognostic indicators and serum $25-\mathrm{OH}$ vitamin D levels. Ann. Surg. Oncol.2012;19:2590-2599.

4. Yao S, Ambrosone CB. Associations between vitamin $\mathrm{D}$ deficiency and risk of aggressive breast cancer in African-American women. J. Steroid Biochem. Mol. Biol. 2013;136:337-341

5. Consolidated Report of Population Based Cancer Registries 2001-2004. National Centre for Disease Informatics and Research National Cancer Registry Programme (Indian Council of Medical Research); 2006.

6. Rakha EA, Reis-Filho JS, Baehner F, et al. Breast cancer prognostic classification in the molecular era: the role of histological grade. Breast Cancer Res 2010; 12: 207.

7. Perou CM, Sorlie T, Eisen MB, et al. Molecular portraits of human breast tumours. Nature 2000; 406: 747-52.

8. Cummings MC, Chamber R, Simpson PT, Lakhani SR. Molecular classification of breast cancer. Is it time to pack up our microscopes? Pathology. 2011;43(1):1-8.

9. Rainville C, Khan Y, Tisman G. Triple negative breast cancer patients presenting with low serum vitamin D levels: a case series. Cases J. 2009;2:8390

10. Ritu G, Gupta A. Vitamin D Deficiency in India: Prevalence, Causalities and Interventions. Nutrients 2014; 6:729-775.

11. Yousef FM, Jacobs ET, Kang PT, Hakim IA, Going S, Yousef JMet al. Vitamin D status and breast cancer in Saudi Arabian women: case-control study. Am. J. Clin. Nutr. 2013;98:105-110.

12. Rakha EA, Ellis IO. Triple-negative/basal-like breast cancer: review. Pathology .2009; 41:40-47.

13. Slamon DJ, Clark GM, Wong SG, Levin WJ, Ullrich A, McGuire RL. Human breast cancer: correlation of relapse and survival with amplification of the HER-2/neu oncogene. Science. 1987;235:177-182.

14. Lukong KE. Understanding breast cancer - The long and winding road. BBA Clinical.2017;7: 64 -77. 
15. Kim HJ, Lee YM, Ko BS, Lee JW, Yu JH, Son BHet al. Vitamin D Deficiency is Correlated with Poor Outcomes in Patients with Luminal-type Breast Cancer Surg. Oncol.2011; $18: 1830-1836$.

16. LaPorta E, Welsh J. Modeling vitamin D actions in triple negative/basal-like breast cancer. J. Steroid Biochem. Mol. Biol. 2014;144:65-73.
17. Gissel T, Rejnmark L, Mosekilde L, Vestergaard P. Intake of vitamin D and risk of breast cancer a meta-analysis. J. Steroid Biochem. Mol. Biol. 2008;111:195-199.

18. Peppone LJ, Huston AJ, Reid ME, Rosier RN, Zakharia Y, Trump DL, et al. The effect of various vitamin D supplementation regimens in breast cancer patients. Breast Cancer Res Treat. 2011;127:171-7.

*Corresponding author:

Dr Sanjeev Garg, H.No.111, sector 46A, Chandigarh

Phone: +91 9878517283

Email: lifeline.sanjeev@gmail.com

Date of Submission : 17.04.2017

Date of Acceptance : 20.07.2017

Financial or other Competing Interests: None.

Date of Publication : 19.12.2017 